\title{
Individuals with unilateral transtibial amputation exhibit reduced accuracy and precision during a targeted stepping task
}

\author{
R.J. Foster ${ }^{\mathrm{a}, *}$, M.R. Haley ${ }^{\mathrm{a}}$, I.W. Talbot ${ }^{\mathrm{b}}$, A.R. De Asha ${ }^{\mathrm{c}, \mathrm{d}}$, C.T. Barnett ${ }^{\mathrm{d}}$ \\ ${ }^{a}$ Research Institute for Sport and Exercise Sciences, Tom Reilly Building, Byrom Street, Liverpool John Moores University, Liverpool L3 3AF, UK \\ ${ }^{\mathrm{b}}$ Pace Rehabilitation Limited, 7 Bredbury Park Way, Bredbury, Stockport, Cheshire SK6 2SN, UK \\ ${ }^{\mathrm{C}}$ C-Motion, Inc., 20030 Century Blvd, Suite 104A Germantown, MD 20874 USA \\ ${ }^{\mathrm{d}}$ School of Science and Technology, Nottingham Trent University, Nottingham NG11 8NF, UK
}

\section{A R T I C L E I N F O}

\section{Article history:}

Accepted 2 April 2020

Available online $\mathrm{xxxx}$

\section{Keywords:}

Transtibial Amputation

Prosthesis User

Foot Placement

Locomotion

Single Limb Support

Stance Phase Stability

\begin{abstract}
A B S T R A C T
Accurate foot placement is important for dynamic balance during activities of daily living. Disruption of sensory information and prosthetic componentry characteristics may result in increased locomotor task difficulty for individuals with lower limb amputation. This study investigated the accuracy and precision of prosthetic and intact foot placement during a targeted stepping task in individuals with unilateral transtibial amputation (IUTAs; $\mathrm{N}=8,47 \pm 13 \mathrm{yrs}$ ), compared to the preferred foot of control participant's $(\mathrm{N}=8,33 \pm 15 \mathrm{yrs})$. Participants walked along a 10-metre walkway, placing their foot into a rectangular floor-based target with dimensions normalised to a percentage of participant's foot length and width; 'standard' $=150 \%$ x 150\%, 'wide' = 150\% x 200\%, 'long' = 200\% x 150\%. Foot placement accuracy (relative distance between foot and target centre), precision (between-trial variability), and foot-reach kinematics were determined for each limb and target, using three-dimensional motion capture. A significant foot-bytarget interaction revealed less mediolateral foot placement accuracy for IUTAs in the wide target, which was significantly less accurate for the intact $(28 \pm 12 \mathrm{~mm})$ compared to prosthetic foot $(16 \pm 14 \mathrm{~mm})$. Intact peak foot velocity $\left(4.6 \pm 0.8 \mathrm{~m} . \mathrm{s}^{-1}\right)$ was greater than the prosthetic foot $\left(4.5 \pm 0.8 \mathrm{~m} . \mathrm{s}^{-1}\right)$ for all targets. Controls were more accurate and precise than IUTAs, regardless of target size. Less accurate and precise intact foot placement in IUTAs, coupled with a faster moving intact limb, is likely due to several factors including reduced proprioceptive feedback and active control during prosthetic limb single stance. This could affect activities of daily living where foot placement is critical, such as negotiating cluttered travel paths or obstacles whilst maintaining balance.
\end{abstract}

(c) 2020 Elsevier Ltd. All rights reserved.

\section{Introduction}

Lower limb amputation has a number of physical effects that reduce individuals' mobility. As individuals regain locomotor function, they must adapt to their altered musculoskeletal system and subsequent sensory changes, as well as the mechanical constraints of the prosthetic devices they use. This leads individuals with lower limb amputation to develop locomotor adaptations (Barnett et al., 2009; Hak et al., 2014). As a result, maintaining balance can be challenging for individuals with lower limb amputation, which is reflected by their increased risk of falling (Miller et al., 2001).

\footnotetext{
* Corresponding author.

E-mail address: R.J.Foster@ljmu.ac.uk (R.J. Foster).
}

The positioning of the foot relative to the body's centre of mass during stance plays a crucial role in maintaining stability during gait (Bruijn and Van Dieën, 2018). The margins of stability concept, which measures locomotor stability using centre of mass and lower limb dynamics (Hof et al., 2005), has been used to reveal that the step length asymmetry reported previously in individuals with unilateral transtibial amputation (IUTAs), may serve a functional purpose in maintaining dynamic stability (Barnett et al., 2009; Hak et al., 2014). This raises the possibility that errors in foot placement could be detrimental to dynamic stability in this population. This may be particularly pertinent when completing activities of daily living (ADLs) where the margin for error in foot placement is small, such as negotiating cluttered travel paths or avoiding and/or stepping over obstacles.

Indeed, when stepping up to or down from a kerb, IUTAs displayed specific lead limb preferences; when stepping down, IUTAs 
tended to lead with their affected limb and when stepping up, tended to lead with their intact limb (Barnett et al., 2014) with authors suggesting that IUTAs utilised the improved capacity (e.g. greater ankle/knee mobility and power generation/absorption) of the intact limb to control these movements. When crossing an obstacle during gait, IUTAs tended to walk more slowly and position their feet closer to the obstacle prior to and after crossing it compared to control participants (Buckley et al., 2013). This appeared to ensure successful toe and heel clearance over the obstacle. Considering that lateral stability is closely related to energetic cost during gait (Bruijn and Van Dieën, 2018; Donelan et al., 2004) and individuals with lower limb amputation have reduced mediolateral stability (Beltran et al., 2014; Gates et al., 2013), foot placement and subsequent dynamic stability, may also have relevance for the increased energetic cost of walking in this population (Gailey et al., 1994).

Despite investigations of locomotor adaptations from a biomechanical perspective, one key issue that remains unexplored is that of targeted foot positioning during ADLs. The combination of changes to the musculoskeletal system, the altered sensory information received by the individual and the prosthetic device mechanical characteristics are likely to negatively influence IUTAs' targeted stepping ability. If established, this may explain some of the reliance on the intact limb during locomotor behaviour and has relevance to falls risk reported in this population. Investigating how the control of the lower limbs prosthetic devices affect the accuracy (an ability to place the foot in the desired location) and precision (the variability of foot placement from one attempt to the next) of foot placement during locomotor tasks would go some way in aiding this understanding. Variability in foot placement can be modulated based on surface area availability, with precise foot placements on a narrow walkway leading to a decrease in stepwidth variability in healthy adults (Verrel et al., 2010). Furthermore, online alterations to the trajectory of the foot when stepping into floor-based targets can improve the accuracy of foot placement in healthy participants (Reynolds and Day, 2005). Thus, the existing evidence base suggests adaptability is desirable during targeted stepping. However, it is not known if and how IUTAs modulate accuracy and precision of foot placement during targeted stepping with either their prosthetic or intact limb. Understanding how well individuals with lower limb amputation are able to perform targeted stepping with the affected and intact limbs has relevance for rehabilitation in terms of the locomotor tasks prescribed and practiced. This also has relevance for prosthetic prescription in terms of device characteristics and their influence on targeted stepping performance. Both of these issues are also likely to feed into an individual's balance ability and thus, their subsequent falls risk.

This study aimed to determine the accuracy and precision of IUTAs' prosthetic and intact foot placement when stepping into a floor-based target, in comparison to control participants' preferred foot placement. It was hypothesised (1) that IUTAs would show increased foot placement error (reduced accuracy and precision) on the intact compared to the prosthetic foot when stepping into a target. This hypothesis was derived from the previously reported reliance on intact limb function during single limb stance during stepping behaviour. This may suggest that the stance limb and its ability to function during single limb support may be related to and reflected in targeted stepping performance. It was also hypothesised (2) that a wider or longer floor-based target would result in increased foot placement error on the intact compared to the prosthetic foot in the medial-lateral and anterior-posterior directions respectively, given the increased margin for error. Finally, it was hypothesised (3) that IUTAs would show increased foot placement error in both feet (prosthetic and intact) when compared to healthy control participants.

\section{Methods}

\subsection{Participants}

Eight healthy IUTAs and eight healthy control participants (Table 1) consented to take part in the study. All IUTAs were categorized as being at least $\mathrm{K} 3$ on the Medicare Functional Classification scale and wore their habitual prosthesis throughout data collection. IUTAs undergoing amputation less than six months previously, or with ongoing medical issues related to the residual limb (e.g. sores or blisters), and those with cardiovascular disorders, neurological, visual or balance impairments were excluded from taking part. The tenets of the Declaration of Helsinki were observed and institutional ethical approval was obtained.

\subsection{Protocol}

Participants walked along a straight 10 -metre walkway at a self-selected speed, placing their foot into a rectangular floorbased target positioned halfway along the walkway (Fig. 1a). IUTAs were asked to accurately place their prosthetic or intact foot in the centre of the target, and control participants were asked to accurately place their preferred foot in the centre of the target only. No guidance was provided regarding which part of the foot should be used to aim for the target centre. Three rectangular floor-based targets with dimensions normalised to a percentage of each participant's foot length and width with shoes on were used (Fig. 1b). The three target sizes were; $150 \%(1) \times 150 \%(w)$ - 'standard', (2) $150 \%$ (l) $\times 200 \%$ (w) - 'wide', (3) 200\% (l) × 150\% (w) - 'long' (Fig. 1b). Target sizes were selected to represent scenarios in ADLs where foot placement is confined to small surface areas and precision is critical to negotiate the environment successfully (e.g. cluttered environments, step/stair treads).

A triangular cluster of three reflective markers (14 mm diameter) were placed on each shoe over the forefoot to track virtual landmarks created by a digitizing wand (C-Motion, Germantown, MD, USA) at the anterior-inferior (toe-tip) and posterior-inferior (heel-tip) point of each shoe. Reflective markers were positioned on each corner of the floor-based target to determine their position within the capture volume. A reflective marker was also positioned on the anterior thoracic trunk segment.

Participants were randomly allocated one of three starting positions that varied by $\pm 25 \mathrm{~mm}$ to begin each trial. This strategy counters the use of somatosensory feedback regarding target location that can be gained when completing multiple trials that are needed to allow comparison of conditions (Chapman, Scally, \& Buckley, 2012). Kinematic data were captured at $100 \mathrm{~Hz}$ using ten infrared cameras (Qualisys, Gothenburg, Sweden) while participants completed three trials of each limb and target condition. Presentation of target size was fully randomised on a trial-by-trial basis for a complete block of prosthetic or intact foot trials ( 9 trials for each side, IUTAs only), and limb order was counterbalanced between participants. Only three trials were used to avoid potential fatigue in IUTAs when completing the protocol.

\subsection{Data analysis}

Marker trajectories were labelled, gap filled, then exported as . c3d files for further analysis in Visual3D (C-Motion, Germantown, MD, USA). All trajectories were smoothed using a bi-pass second order Butterworth low-pass digital filter with a $6 \mathrm{~Hz}$ cut-off.

\subsubsection{Foot placement variables}

Foot placement within the target was determined as the relative distance between the foot centre and target centre when the 
Table 1

Individual participant characteristics, including time since amputation and functional prosthesis for individuals with unilateral transtibial amputation (IUTAs).

\begin{tabular}{|c|c|c|c|c|c|c|c|c|c|c|}
\hline Group & $\begin{array}{l}\text { Gender } \\
(\mathrm{M} / \mathrm{F})\end{array}$ & $\begin{array}{c}\text { Age } \\
\text { (years) }\end{array}$ & $\begin{array}{l}\text { Height } \\
\text { (m) }\end{array}$ & $\begin{array}{l}\text { Mass } \\
(\mathrm{kg})\end{array}$ & $\begin{array}{l}\text { Amputated } \\
\operatorname{limb}(\mathrm{R} / \mathrm{L})\end{array}$ & $\begin{array}{c}\text { Cause of } \\
\text { amputation }\end{array}$ & $\begin{array}{c}\text { Time since amputation } \\
\text { (years) }\end{array}$ & $\begin{array}{l}\text { Functional } \\
\text { prosthesis }\end{array}$ & $\begin{array}{c}\text { Foot } \\
\text { Length }(\mathrm{m})\end{array}$ & $\begin{array}{c}\text { Foot } \\
\text { Width (m) }\end{array}$ \\
\hline \multicolumn{11}{|l|}{ IUTAS } \\
\hline 1 & M & 56 & 1.85 & 105 & $\mathrm{R}$ & Trauma & 2 & Echelon & 0.35 & 0.14 \\
\hline 2 & M & 27 & 1.77 & 79 & $\mathrm{~L}$ & Trauma & 2 & Proflex & 0.32 & 0.14 \\
\hline 3 & M & 32 & 1.81 & 83 & $\mathrm{~L}$ & Trauma & 2 & Proflex & 0.30 & 0.12 \\
\hline 4 & M & 39 & 1.83 & 87 & $\mathrm{~L}$ & Trauma & 3 & Elite blade & 0.34 & 0.13 \\
\hline 5 & $\mathrm{~F}$ & 67 & 1.65 & 54 & $\mathrm{R}$ & Trauma & 41 & Variflex & 0.30 & 0.11 \\
\hline 6 & M & 46 & 1.91 & 107 & $\mathrm{R}$ & Trauma & 2 & Rush foot & 0.35 & 0.14 \\
\hline 7 & M & 56 & 1.79 & 73 & $\mathrm{R}$ & Vascular & 4 & Panthera foot & 0.31 & 0.11 \\
\hline 8 & M & 50 & 1.86 & 100 & $\mathrm{~L}$ & Trauma & 1 & Echelon & 0.31 & 0.12 \\
\hline $\begin{array}{l}\text { Mean } \\
\quad(S D)\end{array}$ & & $\begin{array}{c}47 \\
(13)\end{array}$ & $\begin{array}{c}1.81 \\
(0.08)\end{array}$ & $\begin{array}{c}86 \\
(18)\end{array}$ & & & $\begin{array}{c}7 \\
(14)\end{array}$ & & $0.32(0.02)$ & $0.13(0.01)$ \\
\hline \multicolumn{11}{|l|}{ Controls } \\
\hline 1 & $\mathrm{~F}$ & 24 & 1.73 & 70 & & & & & 0.27 & 0.11 \\
\hline 2 & M & 58 & 1.80 & 80 & & & & & 0.33 & 0.13 \\
\hline 3 & M & 21 & 1.72 & 74 & & & & & 0.33 & 0.13 \\
\hline 4 & M & 24 & 1.78 & 83 & & & & & 0.30 & 0.12 \\
\hline 5 & M & 26 & 1.82 & 76 & & & & & 0.30 & 0.11 \\
\hline 6 & M & 26 & 1.79 & 67 & & & & & 0.30 & 0.11 \\
\hline 7 & M & 56 & 171 & 91 & & & & & 0.30 & 0.12 \\
\hline 8 & $\mathrm{M}$ & 32 & 1.77 & 82 & & & & & 0.31 & 0.12 \\
\hline $\begin{array}{l}\text { Mean } \\
\quad(S D)\end{array}$ & & $\begin{array}{c}33 \\
(15)\end{array}$ & $\begin{array}{c}1.77 \\
(0.04)\end{array}$ & $\begin{array}{c}76 \\
(10)\end{array}$ & & & & & $0.31(0.02)$ & $0.12(0.01)$ \\
\hline
\end{tabular}

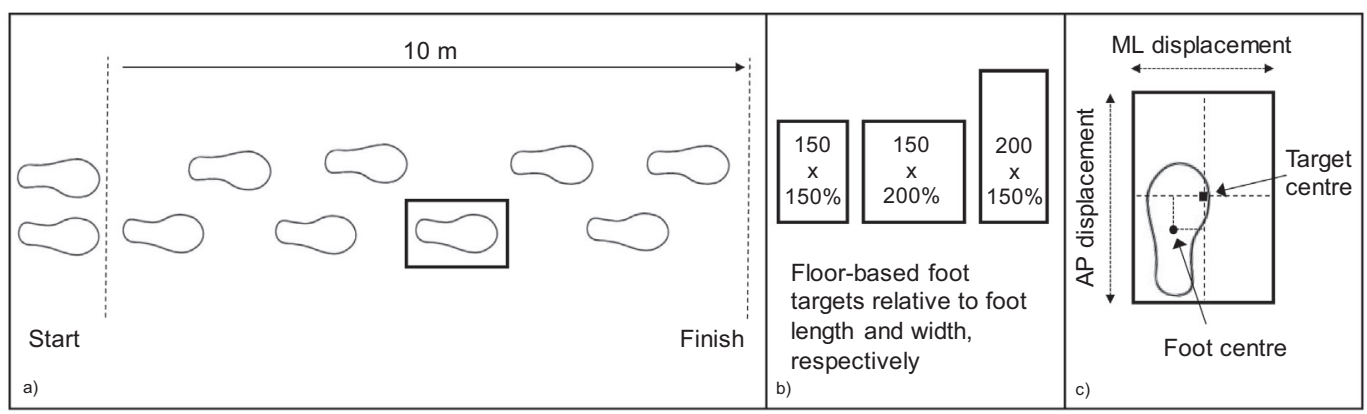

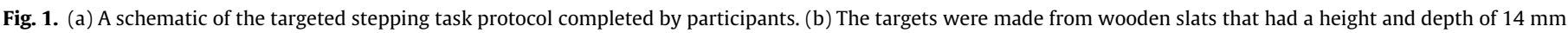

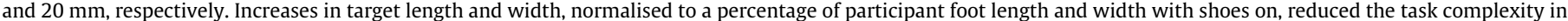

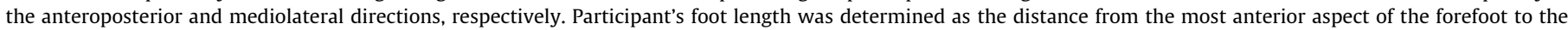

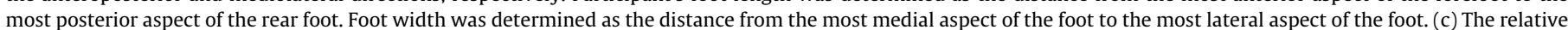

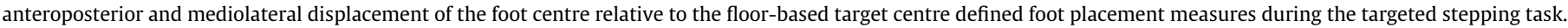

foot was flat inside the target (Fig. 1c). Foot centre was calculated as the mid-point along the vector created between the toe-tip and heel-tip. Target centre was calculated as the mean of the sum of the four anteroposterior and mediolateral reflective marker coordinates positioned on each corner of the target. The following foot placement variables were calculated in the anteroposterior and mediolateral direction separately; Absolute error; the mean scalar foot position distance (regardless of direction) relative to the target centre, reflecting foot placement accuracy. Constant error; the mean vector foot position displacement $( \pm)$ relative to the target, reflecting foot placement bias. Variable error; the variability (one standard deviation) of constant error across trial repetitions, reflecting precision of foot placement (Chapman et al., 2012; Reynolds and Day, 2005). Positive anteroposterior and mediolateral constant error values indicate the foot was positioned anterior and lateral of the target centre, respectively. Larger values reflected increased error across all foot placement variables.

\subsubsection{Stepping kinematics and walking velocity}

Initial foot-reach and terminal foot-reach (Chapman et al., 2012) determined the timing of the foot stepping movement into the target (see Fig. 2), quantifying potential foot trajectory adjustments between foot and target conditions. Approach velocity was calculated as the mean horizontal velocity of the trunk marker, from the initiation of the trial at the beginning of the 10-metre walkway to the instant of touch-down within the target. Walking velocity was calculated over the duration of the whole trial, from start to finish (Fig. 1a).

\subsection{Statistical analysis}

Group mean data were used for statistical analysis. Differences in group characteristics (age, height, mass, foot length, foot width) were analysed using an independent samples $t$-test (SPSS 24.0 for Windows, Chicago, IL, USA). Residual plots were used to visually inspect all variables for normality. Foot placement variables for one control participant were removed for all three target conditions due to outlying data points that exceeded three standard deviations of the remaining group mean.

To address hypotheses (1) and (2), a two-way repeated measures analysis of variance (ANOVA) (SPSS 24.0 for Windows, Chicago, IL, USA) determined differences within IUTAs, with foot (prosthetic and intact) and target size (standard, wide, long) as repeated factors. To address hypothesis (3), we performed two separate two-way mixed design ANOVA analyses; (a) to determine the difference between the prosthetic and control foot for each target 


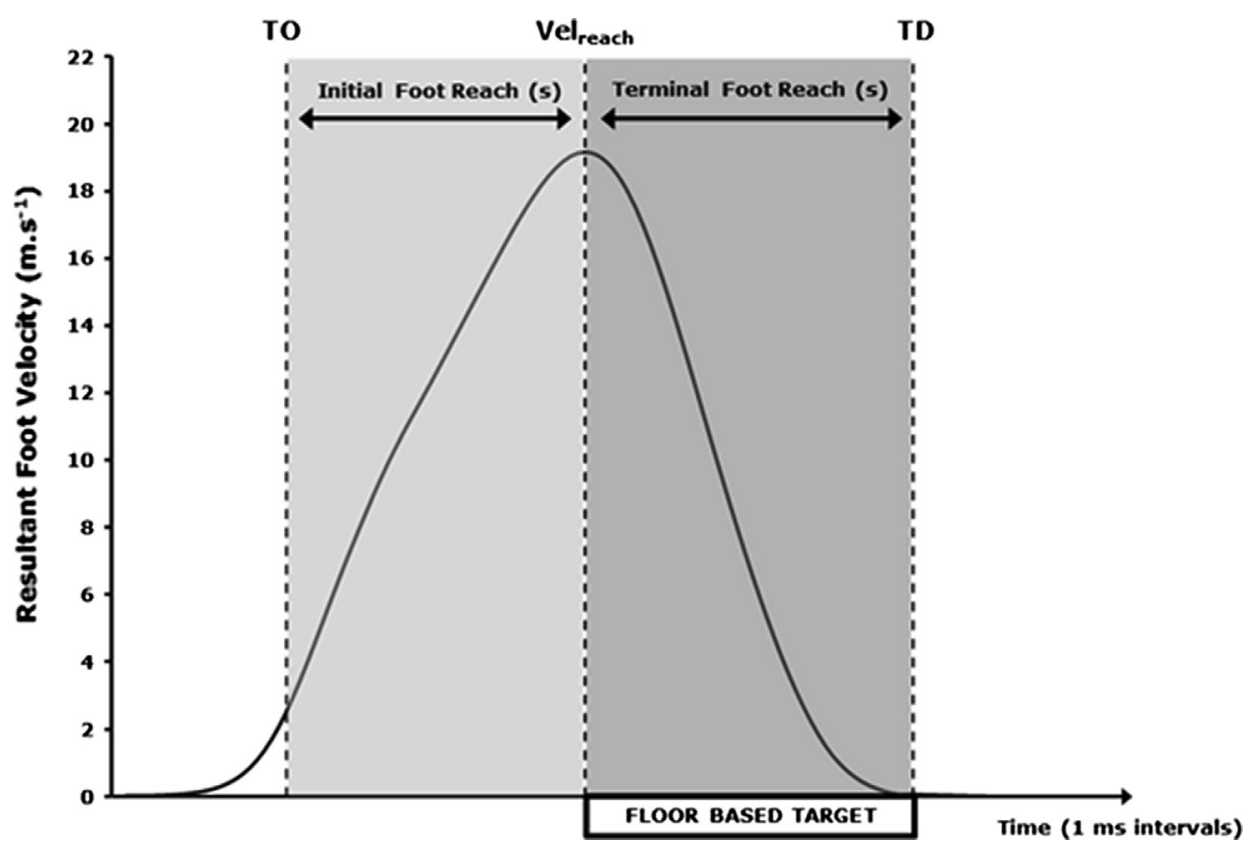

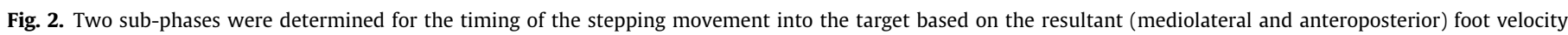

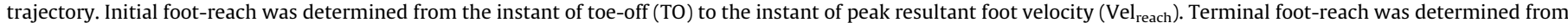

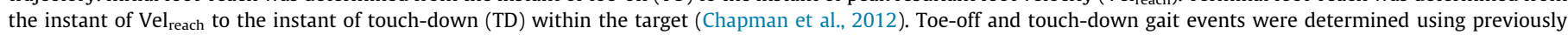
developed kinematic overground gait event detection algorithms (O'Connor et al., 2007).

size, and (b) to determine the difference between the intact and control foot for each target size. Post-hoc analyses were performed using a Bonferroni correction and level of significance was set at $p<0.05$.

\section{Results}

There were no significant differences between the IUTA and control participants based on age $(p=0.083)$, height $(p=0.179)$, mass $(p=0.259)$, foot length $(p=0.106)$ or foot width $(\mathrm{p}=0.192)$ (Table 1$)$. There were no significant differences for approach or walking velocity within or between groups and target size.

\subsection{Intact and prosthetic foot comparisons in IUTAs}

Across all target sizes, intact foot mediolateral absolute error $(18 \pm 12 \mathrm{~mm})$ was increased compared to the prosthetic foot $\left(12 \pm 9 \mathrm{~mm}, \mathrm{~F}_{1,7}=7.104, \mathrm{P}=0.032, \eta_{\mathrm{p}}^{2}=0.504\right.$ ) (Table 2$)$. There were no differences in anteroposterior absolute error or anteroposterior and mediolateral constant and variable error when comparing between the intact and prosthetic feet. Intact foot peak reach velocity $\left(4.6 \pm 0.8 \mathrm{~m} . \mathrm{s}^{-1}\right)$ was greater than the prosthetic foot across all target sizes $\left(4.5 \pm 0.8 \mathrm{~m} . \mathrm{s}^{-1}, \mathrm{~F}_{1,7}=15.909, \mathrm{P}=0.005\right.$, $\eta_{\mathrm{p}}^{2}=0.694$ ), but there were no significant differences in initial or terminal foot reach between feet.

\subsection{Target size manipulation effects on the intact and prosthetic foot in IUTAs}

A significant foot-by-target interaction indicated both prosthetic and intact foot mediolateral absolute error was increased in the wide $(22 \pm 14 \mathrm{~mm})$ compared to the standard $(11 \pm 6 \mathrm{~mm})$ and long target $(12 \pm 6 \mathrm{~mm})$, but the increased absolute error was significantly greater for the intact $(28 \pm 12 \mathrm{~mm})$ compared to the prosthetic foot $\left(16 \pm 14 \mathrm{~mm}, \mathrm{~F}_{2,14}=3.949, \mathrm{P}=0.044\right.$, $\eta_{\mathrm{p}}^{2}=0.361$ ) (Table 2). For all target sizes, IUTAs placed their feet medial of the centre (Fig. 3), but constant error increased when stepping in the wide $(18 \pm 18 \mathrm{~mm})$ compared to the standard $(7 \pm 10 \mathrm{~mm})$ and long target $\left(8 \pm 10 \mathrm{~mm}, \mathrm{~F}_{2,14}=11.709\right.$, $\left.\mathrm{P}<0.001, \eta_{\mathrm{p}}^{2}=0.626\right)$. There were no differences in anteroposterior absolute, constant or variable error, or mediolateral variable error, when comparing between target sizes for both the prosthetic and intact foot. Terminal foot reach was shorter for the wide $(0.241 \pm$ $0.030 \mathrm{~s})$ in comparison to the long target $\left(0.253 \pm 0.031 \mathrm{~s}, \mathrm{~F}_{1.310}\right.$ $\left.9.170=8.395, \mathrm{P}=0.013, \eta_{\mathrm{p}}^{2}=0.545\right)$, but there were no significant differences in initial foot reach and peak reach velocity across target sizes.

\subsection{Comparison between IUTAs and the control group}

Across all target sizes, control foot anteroposterior absolute error was decreased $(20 \pm 9 \mathrm{~mm})$ compared to IUTAs intact $\left(39 \pm 18 \mathrm{~mm}, \mathrm{~F}_{1,14}=12.754, \mathrm{P}=0.003, \eta_{\mathrm{p}}^{2}=0.477\right)$ and prosthetic foot $\left(32 \pm 15 \mathrm{~mm}, \mathrm{~F}_{1,14}=7.045, \mathrm{P}=0.019, \eta_{\mathrm{p}}^{2}=0.335\right)$. Constant error was increased in the anteroposterior direction for IUTAs with both feet significantly overstepping the target centre (intact; $32 \pm 28 \mathrm{~mm}, \mathrm{~F}_{1,14}=5.575, \mathrm{P}=0.033, \eta_{\mathrm{p}}^{2}=0.285$, prosthetic; $\left.27 \pm 20 \mathrm{~mm}, \mathrm{~F}_{1}, 14=6.754, \mathrm{P}=0.021, \eta_{\mathrm{p}}^{2}=0.325\right)$ compared to the control foot $(9 \pm 17 \mathrm{~mm}$ ) (Fig. 3). IUTAs exhibited increased variable error in the anteroposterior direction when placing their intact $\left(22 \pm 10 \mathrm{~mm}, \mathrm{~F}_{1,14}=8.227, \mathrm{P}=0.012, \eta_{\mathrm{p}}^{2}=0.370\right)$ and prosthetic foot $\left(20 \pm 10 \mathrm{~mm}, \mathrm{~F}_{1,14}=5.788, \mathrm{P}=0.031, \eta_{\mathrm{p}}^{2}=0.293\right)$ in the centre of the target compared to the control foot $(14 \pm 9 \mathrm{~mm})$.

A significant foot-by-target interaction indicated that mediolateral absolute error was larger in magnitude for the intact and control foot in the wide $(20 \pm 13 \mathrm{~mm})$ compared to the standard $(11 \pm 5 \mathrm{~mm})$ and long targets $(11 \pm 6 \mathrm{~mm})$, but the increased absolute error in the wide target was significantly greater for the intact

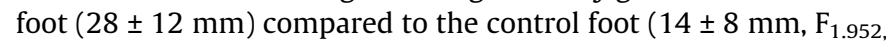
27.324 = 7.410, $\mathrm{P}=0.003, \eta_{\mathrm{p}}^{2}=0.346$ ).

There was a significant foot-by-target interaction effect for mediolateral constant error, whereby the intact and control foot were placed more medial of the target centre for the wide 


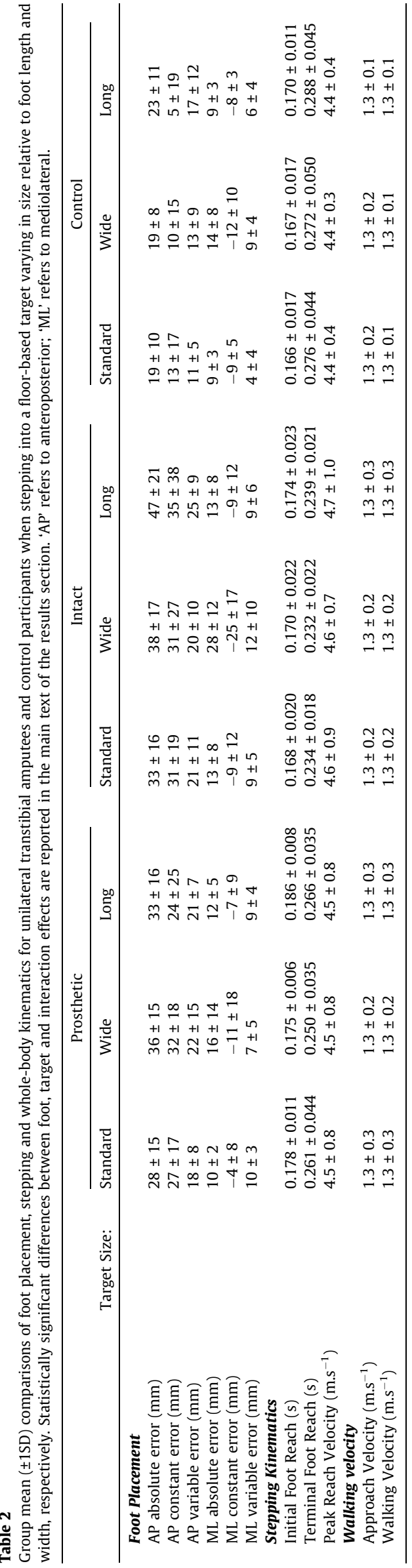

(19 $\pm 15 \mathrm{~mm})$ compared to the standard $(9 \pm 9 \mathrm{~mm})$ and long $(9 \pm 9 \mathrm{~mm})$ target, but intact foot constant error was significantly increased in the wide target $(-25 \pm 17 \mathrm{~mm})$ compared to the control foot $\left(-12 \pm 10 \mathrm{~mm}, \mathrm{~F}_{2,28}=4.985, \mathrm{P}=0.015, \eta_{\mathrm{p}}^{2}=0.263\right)$. IUTAs exhibited increased variable error when placing their intact foot $(10 \pm 7 \mathrm{~mm})$ in the centre of the target compared to the control foot $\left(6 \pm 4 \mathrm{~mm}, \mathrm{~F}_{1,14}=9.379, \mathrm{P}=0.008, \eta \mathrm{p}^{2}=0.401\right)$. There were no significant differences in mediolateral absolute, constant or variable error between the prosthetic and control foot.

Initial foot reach was shorter for the control $(0.168 \pm 0.014 \mathrm{~s})$ compared to the prosthetic foot $\left(0.180 \pm 0.009 \mathrm{~s}, \mathrm{~F}_{1,14}=4.714\right.$, $\mathrm{P}=0.048, \eta_{\mathrm{p}}^{2}=0.252$ ). Initial foot reach was also significantly shorter for the wide $(0.171 \pm 0.013 \mathrm{~s})$ compared to the long target $\left(0.178 \pm 0.012 \mathrm{~s}, \mathrm{~F}_{2,28}=4.795, \mathrm{P}=0.016, \eta_{\mathrm{p}}^{2}=0.255\right)$ for both the control and prosthetic feet. Terminal foot reach was significantly longer for the control $(0.279 \pm 0.045 \mathrm{~s})$ compared to the intact foot $\left(0.235 \pm 0.020 \mathrm{~s}, \mathrm{~F}_{1,14}=6.132, \mathrm{P}=0.027, \eta_{\mathrm{p}}^{2}=0.305\right)$. A main effect of target indicated that terminal foot reach was shorter for the wide $(0.251 \pm 0.039 \mathrm{~s})$ in comparison to the long target for both IUTAs and control participants $(0.264 \pm 0.039 \mathrm{~s}$, prostheticcontrol; $\mathrm{F}_{2,28}=8.497, \mathrm{P}=0.001, \eta_{\mathrm{p}}^{2}=0.378$, intact-control; $\left.\mathrm{F}_{2,28}=4.973, \mathrm{P}=0.014, \eta_{\mathrm{p}}^{2}=0.262\right)$. There were no significant differences in peak reach velocity for all feet and target sizes.

\section{Discussion}

The aim of the current study was to determine the accuracy and precision of IUTAs prosthetic and intact foot placement when stepping into a floor-based target, when compared to control participants. Generally, IUTAs exhibited increased foot placement error (reduced accuracy and precision) when positioning their intact foot into the floor-based target compared to their prosthetic foot and control participants preferred foot.

The hypothesis that (1) IUTAs would show increased foot placement error on the intact compared to the prosthetic foot during targeted stepping, and (2) that a wider or longer floor-based target would result in increased foot placement error on the intact compared to the prosthetic foot were both partially supported. The hypothesis (3) that IUTAs would show increased foot placement error in both limbs (prosthetic and intact) when compared to healthy control participants was supported. Foot placement measures in the anteroposterior direction did not differ between the prosthetic and intact foot of IUTAs but control participants were more accurate and precise than both the prosthetic and intact foot for all target sizes. For the majority of trials IUTAs and control participants overstepped the target centre. On average, the control foot was positioned $\sim 10 \mathrm{~mm}$ and both the prosthetic and intact foot were positioned $\sim 30 \mathrm{~mm}$ anterior of the target centre. Despite previous literature demonstrating that asymmetries exist between limbs in IUTAs during walking, with a decrease in intact step length $(\sim 5 \%)$ and forward foot placement $(\sim 8 \%)$ compared to the prosthetic side (Hak et al., 2014), the present study findings suggest IUTAs are able to modulate anteroposterior foot placement appropriately (i.e. adjust for any asymmetry) in both feet when accuracy and precision are critical in order to negotiate the environment successfully.

There were within- and between-group effects related to mediolateral foot placement. Specifically, absolute and constant mediolateral foot placement error were increased with the intact compared to the prosthetic foot, particularly when stepping into a wide target. All foot placement measures were more accurate and precise for the control foot compared to the intact foot, but not the prosthetic foot. That IUTAs intact foot placement was worse than the prosthetic limb, may be related to the previously reported reliance on the intact limb to control stepping to and from 


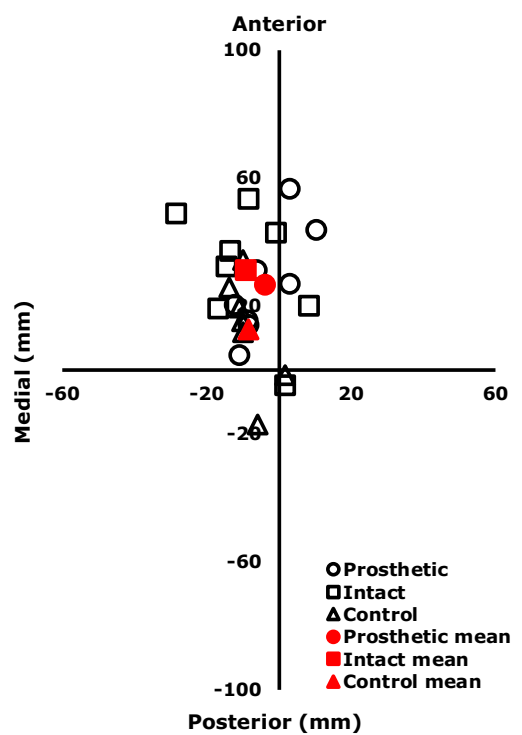

a)

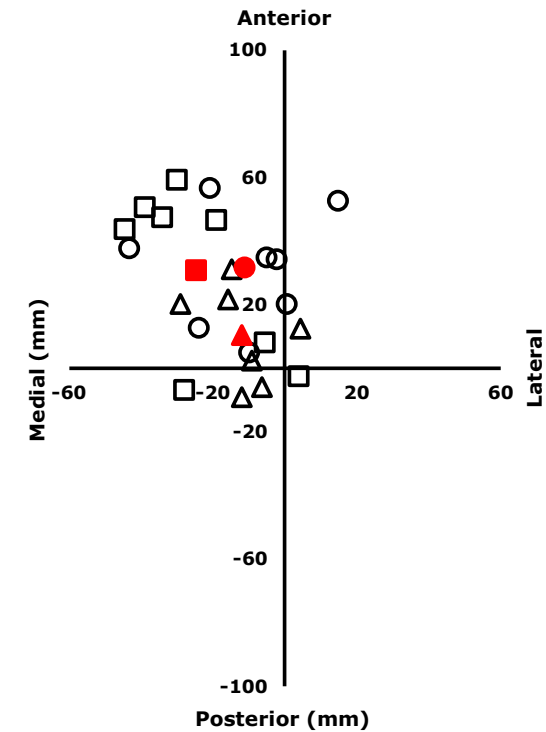

b)

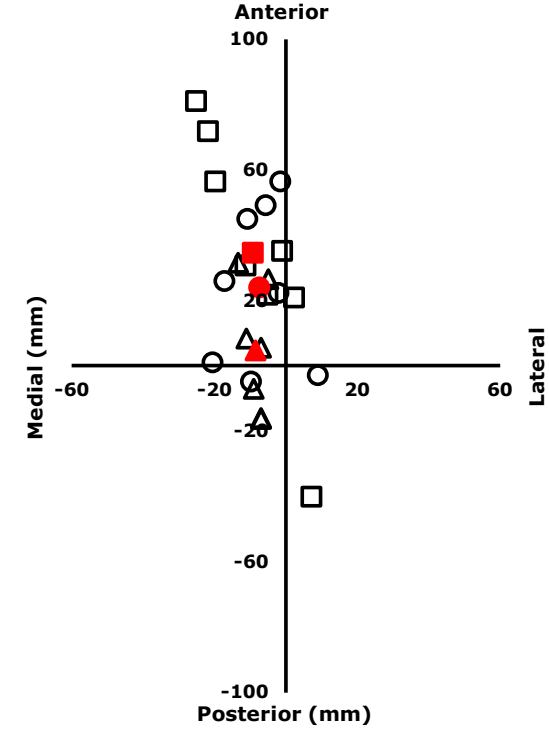

c)

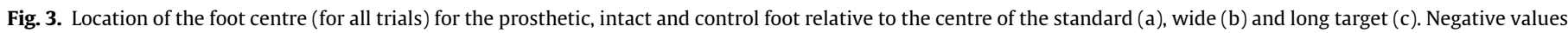
on the horizontal and/or vertical axis indicate that the foot was positioned medial and/or posterior of the target centre, respectively.

a raised surface (Barnett et al., 2014). During single limb support on the affected side, the reduced capabilities of the residual limb and mechanical constraints of the prosthetic device may limit IUTAs in adjusting intact foot placement error. Conversely, intact limb single support may allow for continual, accurate and precise adjustment of affected foot trajectory. Similarly, increased mediolateral foot placement error in the intact limb may relate to well established effects linking gait stability and the energetic cost of walking in IUTAs. Previous research has demonstrated that IUTAs have an increased cost of walking when compared to matched controls (Gailey et al., 1994). This is due to a number of factors including prosthetic componentry (Schmalz et al., 2002), age (Esposito et al., 2014) and comorbidities (Torburn et al., 1995). However, the lateral stability of gait has been shown to be closely related to the energetic cost of walking (Bruijn and Van Dieën, 2018; Donelan et al., 2004) and IUTAs have been shown to have reduced mediolateral gait stability (Beltran et al., 2014; Gates et al., 2013). Therefore, if IUTAs are not able to place their feet accurately and precisely, particularly when using the intact foot, then this may decrease the mediolateral stability of gait, which may subsequently increase the energetic cost of walking. However, this hypothetical link, whilst logical, requires further investigation. A key follow on question is then, what underpins this inability to control foot placement in IUTAs? One explanation may be that given mediolateral stability of gait requires sensory feedback (Donelan et al., 2004), IUTAs foot placement is worse, potentially due to the sensory disruption resulting from amputation surgery. This suggests that the preparation for and adjustments of foot placement during swing, are more easily achieved when in single limb stance on the intact limb. When in prosthetic single limb stance, increased intact foot placement error may result from altered proprioceptive feedback, particularly from the residuum-socket interface and control attributed to the prosthetic limb (Mak et al., 2001). IUTAs tended to move the intact foot towards the target at a faster rate, reflected in greater peak reach velocity for all target sizes. This increase may reflect a desire to initiate intact limb stance as quickly as possible, as a result of prosthetic limb instability. In combination with increased intact foot placement error, a faster moving intact foot suggests that there is a speed-accuracy trade-off when completing the task, whereby faster steps into the floor-based target exhibit greater endpoint error, which is similar to previous findings on visually guided foot-targeting tasks (Chapman et al., 2012; Reynolds and Day, 2005). Although the current study does not present data to show IUTAs are unstable during prosthetic single limb stance, findings clearly relate to previous reports of IUTAs taking longer steps with their prosthetic limb (Barnett et al., 2014; Hak et al., 2014) or a preference to lead with the prosthetic limb when stepping down from a kerb (Barnett et al., 2014). Similarly, the current data showing that as target size increases/widens, foot placement error was increased may reflect IUTAs compromising accuracy and precision of the targeting intact foot to focus more on overall gait function, hence the lack of change in walking speed observed in the current study. IUTAs may therefore modulate their mediolateral intact foot placement less where there is a greater surface area to step in/on, in favour of greater stability by increasing step width. This affect may be problematic in situations where foot placement quality is required and task execution time is reduced e.g. unplanned or reactive side-stepping during locomotion.

\subsection{Limitations}

There are a number of limitations that should be considered when interpreting the results of this study. Firstly, measures of foot placement performance were defined using the geometric centre of the foot. However, it is not clear how participants, particularly IUTAs, conceptualise what part or area of the foot constitutes the centre and how that relates to their locating of the floor-based target. This may be further complicated by the appearance of the prosthetic device and/or footwear worn by participants. As this may explain some of the medial bias observed in the current study, further investigation is required to understand what part of the foot IUTAs use to aim directly towards the floor-based targets. The small number of trials $(n=3)$ used to provide a measure of variable error may not have been sufficient, although increasing the number of trials may have led to fatigue within IUTAs. Given the relationship between foot placement with gait stability, application of a full body biomechanical model in future investigations would enable the accurate calculation of whole-body centre of mass, which could determine whether IUTAs were closer to their 
margins of stability during the foot-targeting task. The sample size for each group of participants was relatively small. However, the paucity of research in this area meant that reliable a priori power analyses were not possible, thus the current findings may inform sample size estimations for similar future studies on targeted stepping in IUTAs (Batterham \& Atkinson, 2005). Although there were no differences in participant characteristics between IUTAs and the control group, future research should aim to match participants by age, to avoid any age effects on balance and gait variability (Schrager et al., 2008). Findings from the current study pertain to relatively active IUTAs. Increased foot placement errors may be further exacerbated in IUTAs who are less mobile (i.e. K2 or below), or for individuals with a higher level of amputation (i.e. unilateral transfemoral amputation). These factors are likely to have a greater impact on tasks where foot accuracy and precision is more challenging, which would highlight the importance of developing relevant foot-targeting assessments (Houdijk et al., 2012) and even interventions that could improve gait adaptability and improve the clinical decision making process.

\section{Conclusion}

IUTAs were less able to produce accurate and precise foot placements with their intact compared to the prosthetic limb. Control participants exhibited better accuracy and precision than the IUTAs intact foot. Our data supplements current knowledge and understanding of strategies used by IUTAs for completing ADLs where foot placement is relevant. The importance of foottargeting assessments and interventions should be explored in a wider variety of locomotor tasks.

\section{Declaration of Competing Interest}

The authors declare that they have no known competing financial interests or personal relationships that could have appeared to influence the work reported in this paper.

\section{References}

Barnett, C., Vanicek, N., Polman, R., Hancock, A., Brown, B., Smith, L., Chetter, I., 2009. Kinematic gait adaptations in unilateral transtibial amputees during rehabilitation. Prosthet. Orthot. Int. 33 (2), 135-147.

Barnett, C.T., Polman, R.C.J., Vanicek, N., 2014. Longitudinal changes in transtibia amputee gait characteristics when negotiating a change in surface height during continuous gait. Clin. Biomech. 29 (7), 787-793.
Batterham, A.M., Atkinson, G., 2005. How big does my sample need to be? a primer on the murky world of sample size estimation. Physical Therapy in Sport 6 (3), $153-163$.

Beltran, E.J., Dingwell, J.B., Wilken, J.M., 2014. Margins of stability in young adults with traumatic transtibial amputation walking in destabilizing environments. J. Biomech. 47 (5), 1138-1143.

Bruijn, S.M., Van Dieën, J.H., 2018. Control of human gait stability through foot placement. J. R. Soc. Interface 15 (143), 20170816.

Buckley, J.G., De Asha, A.R., Johnson, L., Beggs, C.B., 2013. Understanding adaptive gait in lower-limb amputees: insights from multivariate analyses. J. NeuroEng. Rehabil. 10 (1), 98.

Chapman, G.J., Scally, A., Buckley, J.G., 2012. Importance of binocular vision in foot placement accuracy when stepping onto a floor-based target during gait initiation. Exp. Brain Res. 216 (1), 71-80.

Donelan, J.M., Shipman, D.W., Kram, R., Kuo, A.D., 2004. Mechanical and metabolic requirements for active lateral stabilization in human walking. J. Biomech. 37 (6), 827-835.

Esposito, E.R., Rodriguez, K.M., Ràbago, C.A., Wilken, J.M., 2014. Does unilateral transtibial amputation lead to greater metabolic demand during walking. J Rehabil Res Dev 51 (8), 1287-1296.

Gailey, R., Wenger, M., Raya, M., Kirk, N., Erbs, K., Spyropoulos, P., Nash, M.S., 1994. Energy expenditure of trans-tibial amputees during ambulation at self-selected pace. Prosthet. Orthot. Int. 18 (2), 84-91.

Gates, D.H., Scott, S.J., Wilken, J.M., Dingwell, J.B., 2013. Frontal plane dynamic margins of stability in individuals with and without transtibial amputation walking on a loose rock surface. Gait Post. 38 (4), 570-575.

Hak, L., Van Dieën, J.H., Van Der Wurff, P., Houdijk, H., 2014. Stepping asymmetry among individuals with unilateral transtibial limb loss might be functional in terms of gait stability. Phys. Ther. 94 (10), 1480-1488.

Hof, A., Gazendam, M., Sinke, W., 2005. The condition for dynamic stability. J. Biomech. 38 (1), 1-8.

Houdijk, H., van Ooijen, M.W., Kraal, J.J., Wiggerts, H.O., Polomski, W., Janssen, T.W., Roerdink, M., 2012. Assessing gait adaptability in people with a unilateral amputation on an instrumented treadmill with a projected visual context. Phys. Ther. 92 (11), 1452-1460.

Mak, A.F., Zhang, M., Boone, D.A., 2001. State-of-the-art research in lower-limb prosthetic biomechanics-socket interface: a review. J. Rehabil. Res. Dev. 38 (2), $161-174$.

Miller, W.C., Speechley, M., Deathe, B., 2001. The prevalence and risk factors of falling and fear of falling among lower extremity amputees. Arch. Phys. Med. Rehabil. 82 (8), 1031-1037.

O'Connor, C.M., Thorpe, S.K., O'Malley, M.J., Vaughan, C.L., 2007. Automatic detection of gait events using kinematic data. Gait Post. 25 (3), 469-474.

Reynolds, R.F., Day, B.L., 2005. Visual guidance of the human foot during a step. J. Physiol. 569 (2), 677-684.

Schmalz, T., Blumentritt, S., Jarasch, R., 2002. Energy expenditure and biomechanical characteristics of lower limb amputee gait: The influence of prosthetic alignment and different prosthetic components. Gait Post. 16 (3), 255-263.

Schrager, M.A., Kelly, V.E., Price, R., Ferrucci, L., Shumway-Cook, A., 2008. The effects of age on medio-lateral stability during normal and narrow base walking. Gait Post. 28 (3), 466-471.

Torburn, L., Powers, C.M., Guiterrez, R., Perry, J., 1995. Energy expenditure during ambulation in dysvascular and traumatic below-knee amputees: a comparison of five prosthetic feet. J. Rehabil. Res. Dev. 32. 111111.

Verrel, J., Lövdén, M., Lindenberger, U., 2010. Motor-equivalent covariation stabilizes step parameters and center of mass position during treadmill walking. Exp. Brain Res. 207 (1-2), 13-26. 